\title{
Exploring the Factors Influencing the Choice of Oral Care Products: A Review on Personalized Approach
}

\author{
Amit Agrawal, $M D^{1}$ and Ankita Gupta, $M^{2}{ }^{2 *}$ \\ ${ }^{1}$ Associate Professor, Gandhi Medical College \& Hamidia Hospital, Bhopal, MP, India \\ ${ }^{2}$ Senior Lecturer, Public Health Dentistry, Rishiraj College of Dental Sciences, Bhopal, MP, India
}

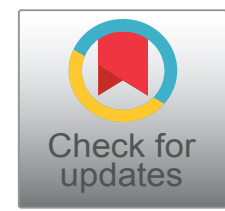

*Corresponding author: Dr. Ankita Gupta, Senior Lecturer, Public Health Dentistry, Rishiraj College of Dental Sciences, Bhopal, MP, India, Tel: +8904838650

\begin{abstract}
Oral care products are the products that are used to cleanse the oral cavity, freshen the breath, and maintain good oral hygiene. Since the dental industry is expanding day by day, numerous types of oral care products are available in the market which makes the selection process very difficult. There are different approaches which influence the choice of oral care products. Among them, personalized approach ranks at the top and should be used by the people to identify the best formulation of oral care that fits into the requirement of the people as proper selection of appropriate oral health care product can play a critical role in improving oral health and also in preventing dental diseases. Hence, the present review was conducted with an aim to explore different factors affecting the choice of oral care products, more specifically with respect to the personalized approach in oral care products consideration.
\end{abstract}

\section{Keywords}

Approach, Dental industry, Oral hygiene, Oral care product

\section{Introduction}

Nowadays, oral health is considered an equally important and integral part of general health. For this reason, the oral cavity is also looked as the 'mirror' of general health. It is known that a healthy mouth and a healthy body go hand in hand. Any deviation in the state of oral health results in poor oral health which can diminish the quality of life of an individual and contribute to the formation of oral diseases. A lot of the factors are responsible for the occurrence of oral diseases like nutritional status, tobacco, smoking, alcohol, poor oral hygiene, and stress. Among these factors, oral hygiene is considered as the most significant factor for the prevention of oral diseases and the preservation of oral health. Maintenance of oral hygiene is the responsibility of both the patient and the dental professional. One of the best methods of improving oral hygiene is by improving oral hygiene practices which can be achieved with the help of oral care products [1].

Oral care products are the products that are used to cleanse the oral cavity, freshen the breath, and maintain good oral hygiene. As the dental industry is expanding day by day, numerous types of oral care products are available in the market which includes toothbrush, toothpaste, mouthwash, floss, and whitening agents but the most widely used oral care products are the toothbrush and toothpaste [2]. Other products are either used by the advice of a dentist or voluntarily. Each one of these agents with the help of their attractive advertisement claims that their product is best. The selection of appropriate oral health care products can play a critical role in improving oral health and also in preventing dental diseases but the most common problem faced by the people is the selection of a right oral care product. Hence, a personalized approach should be used by the people to identify the best formulation of oral care that fits into the requirement of the people and a scheduled repetition of oral care hygiene procedures are a must to obtain the desired results as achieving a healthy mouth takes a lifetime of care.

To the best of our knowledge, no review was published in the past on such a crucial topic and ours is the

Citation: Agrawal A, Gupta A (2020) Exploring the Factors Influencing the Choice of Oral Care Products: A Review on Personalized Approach. Int J Oral Dent Health 6:109. doi.org/10.23937/24695734/1510109

Accepted: June 27, 2020: Published: June 29, 2020

Copyright: (c) 2020 Agrawal A, et al. This is an open-access article distributed under the terms of the Creative Commons Attribution License, which permits unrestricted use, distribution, and reproduction in any medium, provided the original author and source are credited. 
first review conducted with an aim to explore different factors affecting the choice of oral care products, more specifically with respect to personalized approach in oral care products consideration.

\section{Types of Oral Care Products}

Oral care products can be classified in various ways depending upon the method of use, purpose, or composition. a) Based upon the method of their use or action, they are classified as either mechanical (tooth brushing, flossing, and interdental brushing) or chemotherapeutic (chemotherapeutic agents, such as triclosan, chlorhexidine) [3]. Mechanical oral care products include a toothbrush, interdental brush, floss, wooden tips, and chemotherapeutic includes mouthwash, toothpaste, toothpowder, bleaching and whitening agents. b) Based on their purpose of use, they are classified as anti-plaque oral care products and anti-caries oral care products. c) Based on their composition, oral care product can also be classified as natural and synthetic oral care product.

The detailed description of a few commonly used oral care products are:

\section{Toothbrush}

The most widely used oral hygiene aids are toothbrushes and toothpaste. Toothbrushing is a widespread healthcare practice used to remove plaque from teeth, gums, and tongue. Toothbrushes are further classified as manual or electric. There has been controversy regarding the selection of an appropriate toothpaste. Usually, both types of toothbrushes can effectively clean the teeth and the selection of the toothbrush totally depends on the preference of the individual.

\section{Toothpaste}

Toothpaste is either used as paste or gel along with a toothbrush to maintain the health of a tooth. The composition of toothpaste varies based on their purpose but there are some ingredients such as abrasives, fluoride, and detergents that are common to most varieties.

\section{Mouthwash}

They complement oral hygiene measures but cannot replace them. They can be classified into three main types: Antiseptic, plaque-inhibiting, and preventive [4]. Antiseptic mouthwash includes chlorhexidine, plaque-inhibiting mouthwashes include a variety of active ingredients, ranging from antimicrobials (e.g. cetylpyridinium chloride) and agents to prevent bacteria adhering to tooth surfaces (e.g. the amine alcohol delmopinol hydrochloride) to essential oils (e.g. thymol, eucalyptol, and menthol together with methyl salicylate) and preventive mouthwashes are those that contain fluoride for the reversal of early lesions of caries [5]. Apart from having several benefits of antiseptic mouthwash, a few adverse effects have also been noted in some studies. Bescos R, et al. in their clinical study conducted among 36 healthy participants found that mouthwash containing chlorhexidine is associated with a major shift in the salivary microbiome as they increase the abundance of Firmicutes and Proteobacteria, and reduced the content of Bacteroidetes, TM7, SR1, and Fusobacteria. Thus, leading to more acidic conditions and lower nitrite availability in healthy individuals [6].

\section{Factors Influencing the Choice of Oral Care Products}

A number of oral care products are available in the market but the choice of the right oral care products is of great concern. The choice of oral care products depends on several factors. Previous studies done in the past have revealed that the perception of choice of a product is influenced by cultural, social, family, and personal factors.

Social factors that affect the selection of appropriate oral care products include socioeconomic status [7]. Familial factors include education of the individual, the income of the family etc. Previous studies done by Kote $\mathrm{S}$, et al. and Al-Omiri MK, et al. reported a higher percentage of individual that are influenced by the family or parental factors $[8,9]$. Vani $\mathrm{B}$, et al. in their study concluded that in India, the family set up is such that the individual uses the same brand of toothpaste that their entire family uses [10]. A study done by Unanah $\mathrm{AU}$, et al. unfolded the factors that may influence the choice of oral hygiene products and found that out of several factors, income was the major factor influencing the selection of oral hygiene tools [11]. Vani B, et al. found that most of the respondents in their study are influenced by the price of the oral care product then availability, and then packaging [10]. Cultural factors include the tradition of the family regarding using the oral hygiene product. The last factor that may go a long way in determining the choice the oral care product is the personal factors which include age, gender, the influence of the advertisements, perceived performance, previous experience of the individual, awareness by the consumers [12] and personal choices related to the product like taste/lavor, colour, smell, appearance, and content of the products [13]. Among these four factors, personal factors play a vital role in the decision-making of the right oral care product that best fits the individual. A study done by Opeodu OIm, et al. concluded that previous experience seems to be a very strong factor in the choice of both the toothbrush and toothpaste, which suggests that for as long as the respondents are satisfied with a particular product, they will stick to it [14]. The attributes of an oral care product also influence the consumers for decision making [10].

\section{Different Types of Approaches}

\section{Prescriptive approach}

A practitioner may attempt to change a patient's behavior through a prescriptive approach [15]. The pre- 
scriptive approach is not based on the 'felt needs' of the individual, hence found to be quite ineffective in promoting lifelong behavior changes.

\section{Targeted approach}

This approach of selecting oral care products is based upon the felt needs of the individual. The choice is based on the "problem" of an individual instead of the "opinion" of either the dentist or the individual. This includes the inclusion of those oral care products that are meant for specific groups and specific problems.

\section{Personalized approach}

Personalized approach is that in which decisions and treatment are made according to individual patient needs. This approach is based on the patient's choice of selection of oral care products. The literature suggests that by involving the patient in their decision to change will increase the patient's self-efficacy and perceived benefits of the behavior change, thus creating overall improved and lasting oral health [16]. Empowering the patient to understand the cause of the disease process and encouraging him to follow the outcomes enabled him to make a high commitment to a positive behavior change.

\section{Combination}

This includes the combination of all or any two of the approaches.

\section{Personalized Approach in Choosing Oral Care Products}

A personalized approach is based on the decision of the individual. This is the best approach in the selection of oral care products as it best fits the individual. While general recommendations may adequately address the needs of many patients, a dentist may tailor home oral care recommendations to fit the individual patient's needs and wants, focusing on a personalized approach to treatment and prevention.

\section{Personalized approach in selecting toothbrush}

In spite of several types of oral care products in the market, toothbrushing remains the mainstay of oral health measures in the world [17]. According to a survey, out of 6 billion people, 4.2 billion people own a toothbrush globally [18]. Choosing an optimal toothbrush can be a protracted task. The selection of a toothbrush is largely a matter of personal preference, affordability, and professional recommendation. Toothbrushes in the market today are available with special design features of the handle, head, filament that reflects people's understanding of the requirements of effective toothbrushes [19].

Firstly, an individual should choose between a manual and a powered toothbrush. A powered toothbrush is not necessarily superior to a manual toothbrush but they do remove plaque more quickly as compared to a manual toothbrush. Powered toothbrushes can be beneficial for a special group of the individual such as parental brushing of children's teeth; for children and adults who are physically handicapped, mentally retarded, aged, arthritic, or otherwise with poor dexterity; and for those patients who are poorly motivated. These brushes are especially recommended for patients who require a larger handle because powered models are easier to grasp [20]. Studies have reported that a consumers' choice of manual toothbrush depended on the appropriate handle size of the user's age and dexterity and also their appropriate head size to the user's mouth [21]. In a manual toothbrush, an individual is more concerned about the specification of that particular toothbrush i.e. soft, medium, or hard bristles. Usually, persons with wasting diseases and periodontal problems require a soft bristle toothbrush. Otherwise, a medium bristle toothbrush is suitable for all others. In a study conducted by Kote, et al. among the student population in India, the importance of bristle design, and bristle consistency were reported as primary factors influencing their choice for manual toothbrush [8]. The findings of this study are similar to the study by Karve, in which $82.9 \%$ of the population gave importance to the bristles of the manual toothbrush [22]. A study conducted by Opeodu OI, et al. found that the choice of a toothbrush was more influenced by the texture of the bristle than any other factor [14]. Azodo CC, et al. also found that the texture of the toothbrush bristles was the major influencing factor in the choice of a toothbrush [23].

\section{Personalized approach in selecting toothpaste}

Almost all people use toothpaste on a daily basis along with a toothbrush. Toothpaste is an important item in the monthly grocery shopping of almost all persons. As an individual goes to the market, he/she is bombarded with many different brands of toothpaste ranging from different variants of Colgate, Pepsodent, Close-up which comes in a gel form, Anchor which claims itself to be the first vegetarian toothpaste, Dabur, which comes in a powder form and Sensodyne and other fluoridated toothpaste which are basically used for anti-sensitivity purposes [24].

These all brands make numerous different claims about the product but the selection of toothpaste may vary according to the attributes related to the marketing mixes preferred by the consumers. The selection of an appropriate toothpaste should be dependent on its content, quality and efficacy instead of its taste, packaging, advertisement, and brand but unfortunately, most of the individuals make their preferences based on the company name or brand of the product as the endorsement of the product by the celebrity also influences the consumer and affects its decision making. Panigrahi AK in their study revealed that the preference for selecting toothpaste is based on brand of the toothpaste [25]. 
Dani $\mathrm{V}$ in his study found that the brand image, advertising, and sales promotions play an important role in purchasing toothpaste while helping the consumers in comparing with competitor products and selecting the best one [24]. Unanah and Braimoh in their study found that the majority of the individuals (39.3\%) chose their toothpaste based on the cost followed by the taste of the toothpaste (34\%) [11]. Adegbulugbe IC found that the cost, taste, and fluoride content were the major factors influencing the choice of toothpaste [26]. Vani B, et al. conducted a study to understand the external factors that play a role in consumers' choice of toothpaste. The external factors they selected were culture, the price of the product, the quality and other attributes of the product, play in making a toothpaste purchase. The results revealed that $40 \%$ of respondents consider the price when purchasing toothpaste, $40 \%$ of respondents reported that parents had a large impact on the choice of toothpaste. Overall, the researchers concluded that consumers were still less aware than necessary to make proper choices about toothpaste in relation to their oral health needs [10]. Wood L conducted a study with an aim to find those factors that influence an individual's brand-loyal purchasing patterns across six categories of products, one of which was toothpaste. It was found that quality and reputation are the two biggest influences behind toothpaste selection among undergraduate students. Other influential factors for toothpaste choice were the parental influence, as well as gravitating towards the same brand to save time [27]. The results of a study conducted by Shrivastava A also revealed that consumers considered an advertisement as a reliable source of knowledge as compared to others (friend, neighbors, reference group) while buying a toothpaste [28]. These studies conclude to point that the choice of toothpaste is largely determined by other factors rather than its efficacy but this practice needs to be changed and it is not only the duty of dentist but also the society and media persons to create awareness about the selection of an appropriate oral care product.

\section{Personalized approach in selecting mouthwash}

The range of mouthwashes can be bewildering to anyone but it is important to find the right one that suits the needs of the individual. It is important to recognize and remind patients that mouthwash can be a complementary oral care product but cannot replace toothbrush and toothpaste. They can be an alternative to regular oral hygiene procedures when brushing is difficult or cannot be possible for example after an accident or after the placement of sutures after surgery. The question regarding the selection of the right mouthwash is a bit confusing but relevant. The most common reasons found for using mouthwash are for teeth whitening purpose, freshening of breath, preventing dental decay, soothing a dry mouth, and removing sensitivity. The factors responsible for selecting a mouthwash are the primary purpose of use, recommendation by a dentist, and influence by the advertisement [29]. Out of these factors, the preference for selecting a mouthwash should be given to the primary purpose of use and recommendation by a dentist. Macfarlane TV, et al. concluded that the use of mouthwash depends on oral health needs or dentist recommendations [30]. More exposure should be given to the public to enhance public perception and knowledge of mouthwash.

\section{Personalized approach in selecting dental floss}

The inaccessible areas should be cleaned with the help of dental floss but this is purely a matter of consumer choice whether to use it or not. The choice of dental floss is determined by the conditions of the mouth which can differ from person to person. Individuals with maligned teeth do need to use floss but on the advice of a dentist.

\section{Models of Health Behaviour in Support of Per- sonalized Approach}

Several health behavior models have been explored to improve the effectiveness of oral hygiene status. These all health behavior models support the importance of the personalized approach in choosing oral care products and hence in the improvement of oral health. Incorporating behavior science through the use of personal counselling, and motivational interviewing have shown effective in empowering lasting behavior changes [31].

\section{Health belief model (HBM)}

The HBM is an intrapersonal (within the individual, knowledge, and beliefs) theory used in health promotion to design prevention programs. It was designed by Becker and Rosenstock and is one of the best-known models which explores the function of beliefs in decision making [32,33]. According to this model, an individual's beliefs or personalized approach play a vital role in oral health behavior modification. There are three main categories in the model. They are: a) Individual perceptions which are directly related to the knowledge and beliefs that a person has about his behaviours and the outcomes they could have (beliefs that a person has regarding oral care product); b) Modifying factors are those that influence a person's beliefs (the role that advertisement play in modifying a person belief regarding choosing oral care product); c) The likelihood of action is taking appropriate action after weighing out the benefits and the barriers (choosing an appropriate oral care product). Hence, HBM also supports the importance of a personalized approach which consists of internal beliefs and knowledge regarding the prevention of dental diseases or intervention of any oral care program or selection of oral care products.

\section{The theory of reasoned action and planned behav- ior model}

It was given by Ajzen and Fishbein [34]. In the theory 
of reasoned action, the proximal determinant for adopting healthy behaviours is based upon the individual's intention to engage in health-promoting behavior. This theory provides a framework to study attitudes toward behaviours. According to the theory, the most important determinant of a person's behavior is behavior intention. The individual's intention to perform a behavior which is choosing oral care product in the present review is a combination of attitude toward performing the behavior.

\section{Barriers in Choosing Optimal Oral Care Product}

Oral problems although are preventable, remain a major public health problem across the world due to the existence of numerous barriers in oral care delivery and accessibility. These barriers also affect the choice of oral care products. Among these barriers, the biggest barrier is the high cost of oral care products. Although these products are easily available to buy, medium-income people will find it harder to buy these costly products and has a high impact on a regular family's budget. The high cost of oral care products prevents access for the majority of the population to what is considered the ideal method of maintaining a good level of oral hygiene [35]. Other barriers include the inaccessibility of the product in a rural area, low oral health literacy, and lack of awareness [36]. Although there is no scientific evidence to believe that expensive products are better but due to the influence of marketing strategy, advertisements and celebrities, people do tend to believe that the product with the high cost and brand name is the best [35]. A study conducted by Vani B, et al. found that the awareness level of toothpaste brand was less in Bangalore city as people know only the popular brands like Colgate, Pepsodent, and Close-up. So they purchase only known brands [10]. Advertisements have the potential ability to influence consumers into making seemingly more informed purchases, as well as to encourage better at-home oral hygiene practices [37]. This misconception regarding the efficacy of the oral care products need to be removed and their oral health literacy and awareness need to be improved to make each and every oral care product affordable and accessible to everyone.

\section{Conclusion}

Since the personalized approach is the best approach to choose oral care products that rendered clinically significant improvements in patient's oral hygiene and general well-being, an individual should recognize their active role in this crucial decision-making process. By doing so, they can contribute one step towards improving their oral hygiene status.

\section{Consent for Publication}

Not applicable.

\section{Conflict of Interest}

None.

\section{Funding}

None.

\section{Acknowledgments}

None.

\section{References}

1. Sharda A, Sharda J (2010) Factors influencing choice of oral hygiene products used among the population of Udaipur, India. IJDC 2: 1-3.

2. Junaid $A B$, Khan MI, Mansoori MU, Zameer M, Ali SJ (2012) To identify various parameters leading to the growth of dental care products in indian market. IOSRJBM 4: 4-12.

3. Laing E, Ashley P, Gill D, Naini F (2008) An update on oral hygiene products and techniques. Dent Update 35: 270279.

4. (2017) Delivering better oral health: An evidence-based toolkit for prevention. Public health England, London.

5. Varoni E, Tarce M, Lodi G, Carrassi A (2012) Chlorhexidine $(\mathrm{CHX})$ in dentistry: State of the art. Minerva Stomatol 61: 399-419.

6. Bescos R, Ashworth A, Cutler C, Brookes ZL, Belfield L, et al. (2020) Effects of chlorhexidine mouthwash on the oral microbiome. Scientific Reports 10.

7. Martins CC, Oliveira MJ, Pordeus IA, Cury JA, Paiva SM (2011) Association between socioeconomic factors and the choice of dentifrice and fluoride intake by children. Int J Environ Res Public Health 8: 4284-4299.

8. Kote S, Dadu M, Sowmya AR, Aruna DS, Arora D (2013) Knowledge, attitude and behaviour for choosing oral hygiene aids among students of management institutes, Ghaziabad, India. West Indian Med J 62: 758-763.

9. Al-Omiri MK, Al-Wahadni AM, Saeed KN (2006) Oral health attitudes, knowledge among school children in North Jordan. J Dent Educ 70: 179-187.

10. Vani B, Babesh Ganu M, Panchanatham N (2010) Toothpaste brands-a study of consumer behaviour in Bangalore City. JEBS 1: 27-39.

11. Unanah AU, Braimoh OB (2017) Oral hygiene practices and factors influencing the choice of oral hygiene materials among undergraduate students at the University of Port Harcourt, Rivers State, Nigeria. J Dent Allied Sci 6: 3-7.

12. Creusen MEH, Schoormans $J$ (2005) The different roles of product appearance in consumer choice. J Prod Innov Manag 22: 63-81.

13. Sarker S, Yousuf S, Monzoor MZ (2013) Influences on brand selection decisions of staple goods: A study on toothpaste users of Khulna city. J World Econ Res 2: 58-66.

14. Opeodu OI, Gbadebo SO (2017) Factors influencing choice of oral hygiene products by dental patients in a Nigerian teaching hospital. Ann IB Postgrad Med 15: 51-56.

15. Yevlahova D, Satur J (2009) Models for individual oral health promotion and their effectiveness: A systematic review. Aust Dent J 54: 190-197.

16. Silness J, Löe H (1964) Periodontal disease in pregnancy II. Correlation between oral hygiene and periodontal condition. Acta Odontol Scand 22: 121-135.

17. Moynihan P, Petersen PE (2004) Diet, nutrition and the prevention of dental diseases. Public Health Nutr 7: 201-226. 
18. Turner J (2016) Are there really more mobile phone owners than toothbrush owners?

19. Sowinski J, Petrone DM, Wachs GN, Chaknis P, Kemp J, et al. (2008) Efficacy of three toothbrushes on established gingivitis and plaque. Am J Dent 21: 339-345.

20. Balasubramaniam A, Parangimalar Diwakar MK, Brinda B (2017) Factors influencing selection of manual toothbrush among an urban population in Chennai City, India: A cross-sectional study. J Indian Assoc Public Health Dent 15: 388-391.

21. Frandsen $A$ (1986) Mechanical oral hygiene practices. In: Loe H, Kleinman DV, Dental plaque control measures andoral hygiene practices. Oxford, Washington DC, 93-116.

22. Karve S (2011) National monthly refereed journal of research in commerce and management. Abhinav Publ 1 : 103-115.

23. Azodo CC, Ehizele AO, Umoh A, Ojehanon PI, Akhionbare O, et al. (2010) Tooth brushing, tongue cleaning and snacking behaviour of dental technology and therapist students. Libyan J Med.

24. Dani V (2013) Buying behavior of toothpaste in Urban India: A study on Pune city. Pacific Business Review International 5: $48-56$.

25. Panigrahi AK (2015) Brand usership of toothpaste buyers: A study on berhampur city. JRCM 4: 46-54.

26. Adegbulugbe IC (2007) Factors governing the choice of dentifrices by patients attending the dental centre, Lagos University Teaching Hospital. Nig Q J Hosp Med 17: 18-21.

27. Wood L (2008) Dimensions of brand purchasing behavior: Consumers in the 18-24 age group. Journal of Consumer Behavior 4: 9-24.
28. Shrivastava A (2014) Do advertisements work? A study on change in consumers' purchasing behavior due to advertisements. JRMT 3: 14-21.

29. Wilson N, Patel R. Gallagher J, Chapple ILC (2014) How to select the right mouthwash. The Pharmaceutical Journal 292: 1-3.

30. Macfarlane TV, Kawecki MM, Cunningham C, Bovaird I, Morgan R, et al. (2011) Mouthwash use in general population: Results from adult dental health survey in grampian, Scotland. J Oral Maxillofac Res 1.

31. Wilder RS, Bray KS (2000) Improving periodontal outcomes: Merging clinical and behavioral science. Periodontology 2000 71: 65-81.

32. Becker MH (1974) The health belief model and personal health behavior. Health Education Monographs 2: 324-508.

33. Rosenstock IM (1974) Historical origins of the health belief model. Health Education Monographs 2: 328-335.

34. Ajzen I, Fishbein M (1980) Understanding attitudes and predicting social behaviour.

35. Dutra TTB, da Silva ALR, Cabral AM, Neto JMM, Prador RR (2015) The impact of the cost of oral hygiene products on the domestic budget in the urban region of a midsize Brazilian capital city. Rev Gaúch Odontol 63: 13-18.

36. McCutchen, Allison E (2015) Barriers to care and buyer behavior in relation to oral hygiene. Honors college capstone experience/thesis projects.

37. Smith B (2009) The power of the product $p$, or why toothpaste is so important to behavior change. Social Marketing Quarterly 15: 98-106. 